\title{
Wolf-Rayet stars in I Zw 18
}

Francoid Legrand ${ }^{1}$, Daniel Kunth ${ }^{1}$, Jean-René Roy ${ }^{2}$, J. Miguel Mas-Hesse ${ }^{3}$, and Jeremy R. Walsh ${ }^{4}$

${ }^{1}$ Institut d'Astrophysique de Paris, CNRS, 98bis boulevard Arago, F-75014 Paris, France

${ }^{2}$ Dépratement de Physique, Université Laval, Quebec, $P Q$ G1K 74, Canada

${ }^{3}$ LAEFF-INTA, P.O. Box 50727, E-28080 Madrid, Spain

${ }^{4}$ European Southern Observatory, Karl-Schwarzschild-Strasse 2, D-85748 Garching, Germany

\begin{abstract}
Wolf-Rayet stars have been detected in the NW region of the metal-poor starburst galaxy IZw 18. The integrated luminosity and FWHM of the bumps at $4650 \AA$ and $5808 \AA$ are consistent with the presence of a few individual stars of WC4 or WC5 type. The unexpected presence of WC stars in such a low-metallicity galaxy could, however, be explained by high mass loss rates, or alternatively favor a binary channel for WR formation. WC stars could also account for the strong and narrow He II $4686 \AA$ emission line which peaks co-spatially with the WR bump emission (see Schaerer 1996).
\end{abstract}

\section{Introduction}

$\mathrm{IZw} 18$ is known to be the most metal-deficient object among the blue compact dwarf galaxies (BCDs), with a metallicity of $1 / 40$ th of the solar value and undergoing a strong star formation event. Moreover, this galaxy presents a strong He II $4686 \AA$ narrow emission line.

\section{Observations and discussion}

Seventeen exposures of 3000 seconds each of the BCD IZw 18 were obtained with the $3.6 \mathrm{~m} C F H T$. A long slit (1".52 wide) was used with a position angle of $45^{\circ}$, covering the spectral range $3700-6900 \AA$. A complete description of the observations can be found in Legrand et al. (1997). We found two faint broad emission features, typical of WC stars, around $4650 \AA$ and near $5812 \AA$ (Fig. 1). Similar features have been observed by Izotov et al. (1998) with a different slit position.

Using the models of Stasińska \& Leitherer (1996), we checked that these bumps were not due to narrow nebular emission lines such as CIII $4650 \AA$, O II $4651 \AA$, [FeIII] $4658 \AA$, [ArIv] 4711,4740 $\AA$ and N III 4634,4640 $\AA$. After subtraction of the expected nebular lines, the residual bumps appears centered at $4645 \AA$ and $5820 \AA$ with fluxes of respectively $9.90( \pm 3) \times 10^{36} \mathrm{erg} \mathrm{s}^{-1}$ and $4( \pm 1.5) \times 10^{36} \mathrm{erg} \mathrm{s}^{-1}$. The calibration of Smith (1991), indicates that these 

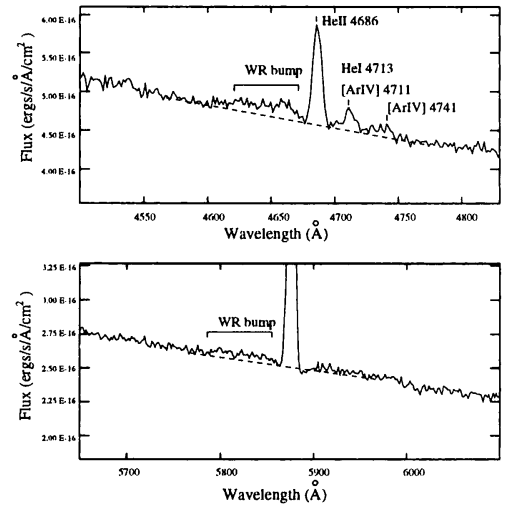

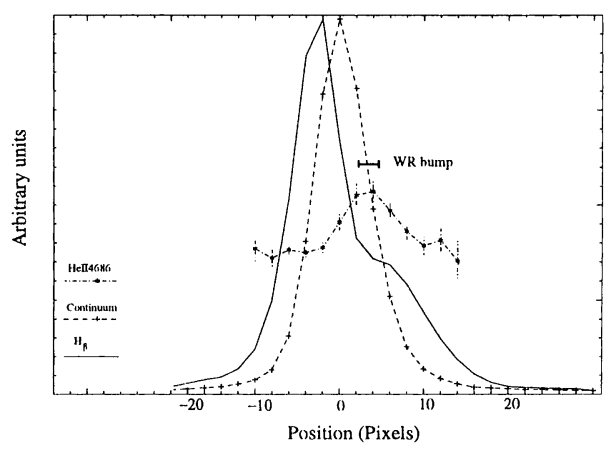

Figure 1. Left: Regions of the spectrum of I Zw 18 around the He II $4686 \AA$ (upper) line and the He I $5876 \AA$ (lower). The spectrum is integrated over $77^{\prime \prime} 8$ centered on the maximum continuum emission. The broken line shows the position of the fitted continuum.

Figure 2. Right: Spatial location of the different spectral emission features along the slit $\left(1\right.$ pixel $\left.=00^{\prime} 3145\right)$. Position of the maximum emission in the WRbump is indicated with bold line.

fluxes are compatible with the presence of one or two WC4 or WC5 stars, and no WN. However, we cannot exclude the presence of $\mathrm{WN}$ in $\mathrm{IZw} 18$ at other positions, as discussed by De Mello et al. (1998).

Moreover, we find that the bumps at $4645 \AA$ and $5820 \AA$ are correlated in position with the maximum of the nebular emission line He II $4686 \AA$ relative to $\mathrm{H} \beta$ (Fig. 2). This indicates, as suggested by Schaerer (1996), that WR stars can be responsible for the nebular emission line He II $4686 \AA$.

Finally, the presence of WR stars in such a low-metallicity galaxy indicates, that mass loss rates may be twice higher than the standard value adopted for very low metallicities, or that the binary channel is an important process of WR stars formation.

\section{References}

De Mello, D.F., Schaerer, D., Heldman, J., Leitherer, C. 1998, ApJ 507, 199

Izotov, Y., Foltz, C.B., Green, R.F., Guseva, N.G., Thuan, T.X. 1997, ApJ 487, L37

Legrand, F., Kunth, D., Roy, J.-R., Mas-Hesse, J.M., Walsh J.R. 1997, A\&A 326, L17

Schaerer, D. 1996, ApJ 467, L17

Smith, L.F. 1991, in: K.A. van der Hucht \& B. Hidayat (eds.), Wolf-Rayet Stars and Interrelations with Other Massive Stars in Galaxies, Proc. IAU Symp. No. 143 (Dordrecht: Kluwer), p. 601

Stasiñska, G., Leitherer, C. 1996, ApJS 107, 661 